\section{International Scientific Journal Theoretical \& Applied Science}

p-ISSN: $2308-4944$ (print) e-ISSN: 2409-0085 (online)

Year: $2014 \quad$ Issue: $10 \quad$ Volume: 18

Published: $30.10 .2014 \quad$ http://www.T-Science.org

SECTION 2. Applied mathematics. Mathematical modeling.
Alexandr Nikolayevich Shevtsov candidate of technical sciences,

member of Publishers International Linking Association (USA), corresponding member of the Kazakhstan National Academy of Natural Sciences, president of International Academy of TAS (USA,

Sweden, Kazakhstan), Associate Professor of Department of Mathematics, deputy director on

Science of faculty of Information Technologies, Automation and Telecommunications, Taraz state University named after M.Kh. Dulati, Kazakhstan Shev AlexXXXX@mail.ru

Gulzhayna Kaliyeva researcher, Kazakhstan k-pop_98@mail.ru

\title{
SOME ASPECTS OF MODELING THE STRANGE ATTRACTORS OF LORENZ IN DELPHI
}

Abstract: This article discusses some of the issues of construction of algorithms in Delphi for solving systems of differential equations with three variable coefficients by the numerical method.

Key words: attractor, modeling, system of differential equations.

Language: Russian

Citation: Shevtsov AN, Kaliyeva G (2014) SOME ASPECTS OF MODELING THE STRANGE ATTRACTORS OF LORENZ IN DELPHI. ISJ Theoretical \& Applied Science 10 (18): 68-81. doi: http://dx.doi.org/10.15863/TAS.2014.10.18.16

\section{О НЕКОТОРЫХ АСПЕКТАХ МОДЕЛИРОВАНИЯ СТРАННЫХ АТТРАКТОРОВ ЛОРЕНЦА НА DELPHI}

Аннотация: В данной статье рассматриваются некоторые вопросы построения алгоритмов на языке Delphi для решения системы дифференщиальных уравнений с тремя переменными коэффициентами численным методом.

Ключевые слова: аттрактор, моделирование, система дифференциальных уравнений.

Исследование классификаций аттракторов представляет особый интерес для различных физических и математических моделей, описывая в отдельных случаях непредсказуемое на первый взгляд поведение динамической системы.

Странный аттрактор, в отличие от обычного имеет два существенных отличия [1, 5-7]:

- траектория такого аттрактора непериодическая (она не замыкается)

- режим функционирования неустойчив (малые отклонения от режима нарастают).

Примерами подобных аттракторов - могут являться: аттрактор Лоренца, аттрактор Рёсслера, соленоид Смейла-Вильямса.

Основным критерием хаотичности аттрактора является экспоненциальное нарастание во времени малых возмущений. Следствием этого - является «перемешивание» в системе, непериодичность во времени любой из координат системы, сплошной спектр мощности и убывающая во времени автокорреляционная функция. Динамика на странных аттракторах часто бывает хаотической [8-10]: прогнозирование траектории, попавшей в аттрактор, затруднено, поскольку малая неточность в начальных данных через некоторое время может привести к сильному расхождению прогноза с реальной траекторией. Непредсказуемость траектории в детерминированных динамических системах называют динамическим хаосом, отличая его от стохастического хаоса, возникающего в стохастических динамических системах. Это явление также называют эффектом бабочки, подразумевая возможность преобразования слабых турбулентных потоков воздуха, вызванных взмахом крыльев бабочки в одной точке планеты в мощное торнадо на другой её стороне вследствие многократного их усиления в атмосфере за некоторое время.

Среди странных аттракторов встречаются такие, хаусдорфова размерность которых отлична от топологической размерности и является дробной. Одним из наиболее известных среди подобных аттракторов является аттрактор Лоренца. 
Impact Factor ISRA (India) $=\mathbf{1 . 3 4 4}$

Impact Factor ISI (Dubai, UAE) $=\mathbf{0 . 3 0 7}$

based on International Citation Report (ICR)
Impact Factor JIF $=1.500$

Impact Factor GIF (Australia) $=\mathbf{0 . 3 5 6}$

Impact Factor SIS (USA) $=\mathbf{0 . 4 3 8}$
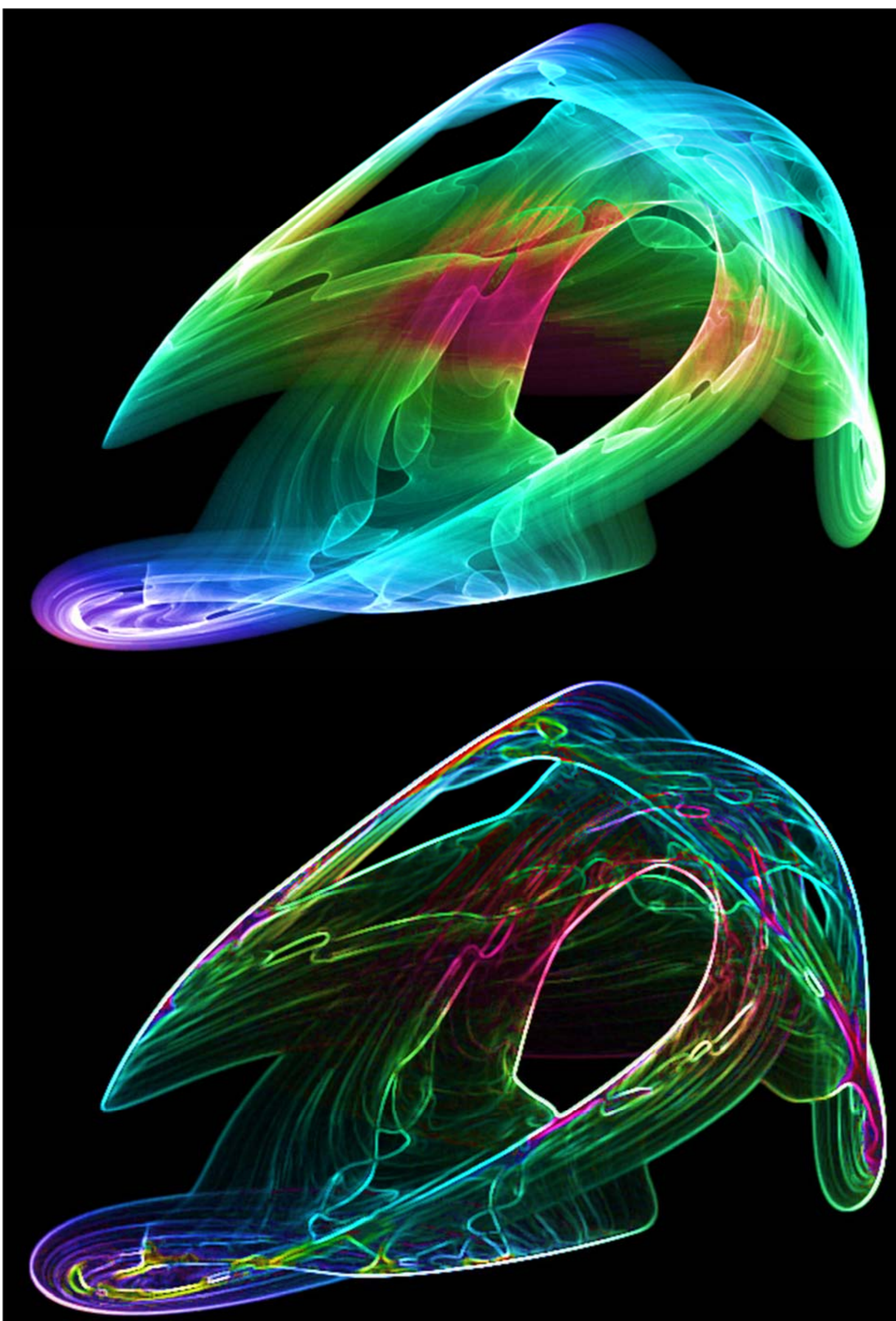

Рисунок 1 - Attractor Poisson Saturne [2]. 
Система дифференциальных уравнений, решение которой, приводит к созданию аттрактора Лоренца имеет вид:

$$
\left\{\begin{array}{l}
\dot{x}=\sigma(y-x) \\
\dot{y}=x(r-z)-y \\
\dot{z}=x y-b^{*} z
\end{array}\right.
$$

Введем обозначения:

$M\left(x_{0}, y_{0}, z_{0}\right)$ - начальная точка,

$a=\sigma$,

$b=r, \quad$ - коэффициенты системы.

$c=b^{*}$

Рассмотрим численное решение этой системы на промежутке

$$
[-10,10]
$$

Разобьем интервал (2) на отдельные промежутки с шагом $h=0.0001$.

Зададим цикл на данном промежутке (2) с шагом $h$, и начиная с точки $M$, будем рассчитывать последовательно все последующие значения системы (1). Получим выражение (3), здесь на каждом последующем шаги рассчитывается очередная точка решения системы (1) численным методом.

$$
\begin{aligned}
& x_{i+1}=x_{i}+a\left(-x_{i}+y_{i}\right) h, \\
& y_{i+1}=y_{i}+\left(b x_{i}+y_{i}-z_{i} x_{i}\right) h, \\
& z_{i+1}=z_{i}+\left(-c z_{i}+x_{i} y_{i}\right) h .
\end{aligned}
$$

Разработаем алгоритм для расчета на языке Delphi[14-28]

$$
\begin{aligned}
& \mathrm{nn}:=-10 ; \\
& \text { while } \mathrm{nn}<10 \text { do } \\
& \text { begin } \\
& \text { nn:=nn+dt; } \\
& \quad \mathrm{x} 1:=\mathrm{x}+\mathrm{a}^{*}(-\mathrm{x}+\mathrm{y})^{*} \mathrm{dt} ; \\
& \mathrm{y} 1:=\mathrm{y}+\left(\mathrm{b}^{*} \mathrm{x}-\mathrm{y}-\mathrm{z}^{*} \mathrm{x}\right) * \mathrm{dt} ; \\
& \mathrm{z} 1:=\mathrm{z}+\left(-\mathrm{c}^{*} \mathrm{z}+\mathrm{x} * \mathrm{y}\right)^{*} \mathrm{dt} ; \\
& \mathrm{x}:=\mathrm{x} 1 ; \\
& \mathrm{y}:=\mathrm{y} 1 ; \\
& \mathrm{z}:=\mathrm{z} 1 ;
\end{aligned}
$$

glcolor3f(Col2, Col2 shr 8+0.1,Col2 shr 16+0.1); glVertex3d(x/10,y/10,z/10-1.2); // точка end;

Отрисовку будем осуществлять с использованием библиотеки OpenGL в 3D формате. Получим следующий результат (Рис.2). Полученная модель позволяет исследовать трехмерный фазовый портрет решения системы дифференциальных уравнений, визуализировать трехмерное решение, находить решение и исследовать при различных начальных условиях и значениях коэффициентов.

Аттрактор Лоренца был найден в численных экспериментах Лоренца, исследовавшего поведение траекторий нелинейной системы: при следующих значениях параметров: $\sigma=10, r=28$, $b=8 / 3$. Эта система вначале была введена как первое нетривиальное галёркинское приближение для задачи о конвекции морской воды в плоском слое, чем и мотивировался выбор значений $\sigma, r$ и $b$, но она возникает также и в других физических вопросах и моделях[8-13]:

- конвекция в замкнутой петле;

- вращение водяного колеса;

- модель одномодового лазера;

- диссипативный осциллятор с инерционной нелинейностью.

Обозначим физический смысл переменных и параметров в системе уравнений применительно к упомянутым задачам.

Конвекция в плоском слое.

Здесь $x$ отвечает за скорость вращения водяных валов, $y$ и $z$ - за распределение температуры по горизонтали и вертикали, $r$ нормированное число Рэлея, $\sigma$ - число Прандтля (отношение коэффициента кинематической вязкости к коэффициенту температуропроводности), $b$ содержит информацию о геометрии конвективной ячейки.

Конвекция в замкнутой петле.

Здесь $x$ - скорость течения, $y$ - отклонение температуры от средней в точке, отстоящей от нижней точки петли на $90^{\circ}, z-$ то же, но в нижней точке. Подведение тепла производится в нижней точке.

Вращение водяного колеса.

Рассматривается задача о колесе, на ободе которого укреплены корзины с отверстиями в дне. Сверху на колесо симметрично относительно оси вращения льётся сплошной поток воды. Задача равнозначна предыдущей, перевернутой «вверх ногами», с заменой температуры на плотность распределения массы воды в корзинах по ободу.

Одномодовый лазер.

Здесь $x$ - амплитуда волн в резонаторе лазера, $y$ - поляризация, $z$ - инверсия населённостей энергетических уровней, $b$ и $\sigma-$ отношения коэффициентов релаксации инверсии и поля к коэффициенту релаксации поляризации, $r$ - интенсивность накачки.

\section{Поведение решения системы}

Рассмотрим изменения в поведении решения системы Лоренца при различных значениях параметра r. На иллюстрациях приведены результаты численного моделирования для точек с начальными координатами $(10,10,10)$ и $(-10,-$ $10,10)$. 

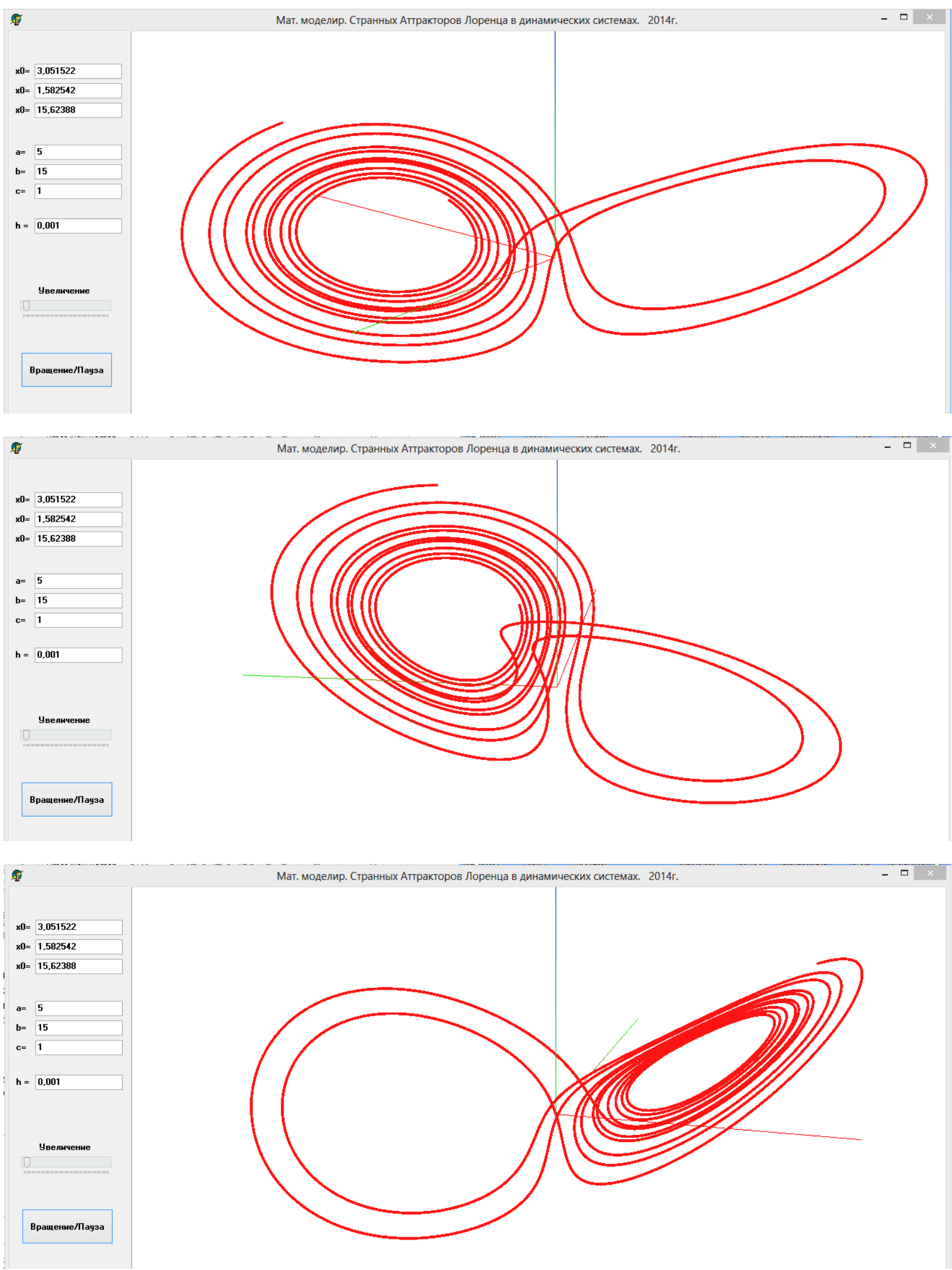

Рисунок 2 - Компьютерная реализация разработанной математической модели странного аттрактора Лоренца. 
$\boldsymbol{r}<\mathbf{1}$ - аттрактором является начало координат, других устойчивых точек нет.

$\mathbf{1}<\boldsymbol{r}<\mathbf{1 3 , 9 2 7}$ - траектории спирально приближаются (это соответствует наличию затухающих колебаний) к двум точкам, положение которых определяется формулами:

Эти точки определяют состояния стационарного режима конвекции, когда в слое формируется структура из вращающихся валов жидкости.

$\boldsymbol{r} \approx \mathbf{1 3 , 9 2 7}$ - если траектория выходит из начала координат, то, совершив полный оборот вокруг одной из устойчивых точек, она вернется обратно в начальную точку - возникают две гомоклинические петли. Понятие гомоклинической траектории означает, что она выходит и приходит в одно и то же положение равновесия.

$r>13,927$ - в зависимости от направления траектория приходит в одну из двух устойчивых точек. Гомоклинические петли перерождаются в неустойчивые предельные циклы, также возникает семейство сложно устроенных траекторий, не являющееся аттрактором, а скорее наоборот, отталкивающее от себя траектории. Иногда по аналогии эта структура называется «странным репеллером» (англ. to repel отталкивать).

$\boldsymbol{r} \approx \mathbf{2 4 , 0 6}$ - траектории теперь ведут не к устойчивым точкам, а асимптотически приближаются к неустойчивым предельным циклам - возникает собственно аттрактор Лоренца. Однако обе устойчивые точки сохраняются вплоть до значений $r \approx 24,74$.

При варьировании $\sigma, r, b$, т.е. $x_{0}+\Delta h, y_{0}+\Delta h, z_{0}+\Delta h \quad$ где $\quad \Delta h=1 \ldots 10$ получим следующие фазовые портреты - Рис.3-6.

Исследование аттрактора при различных значениях $\sigma$.

Таблица 1

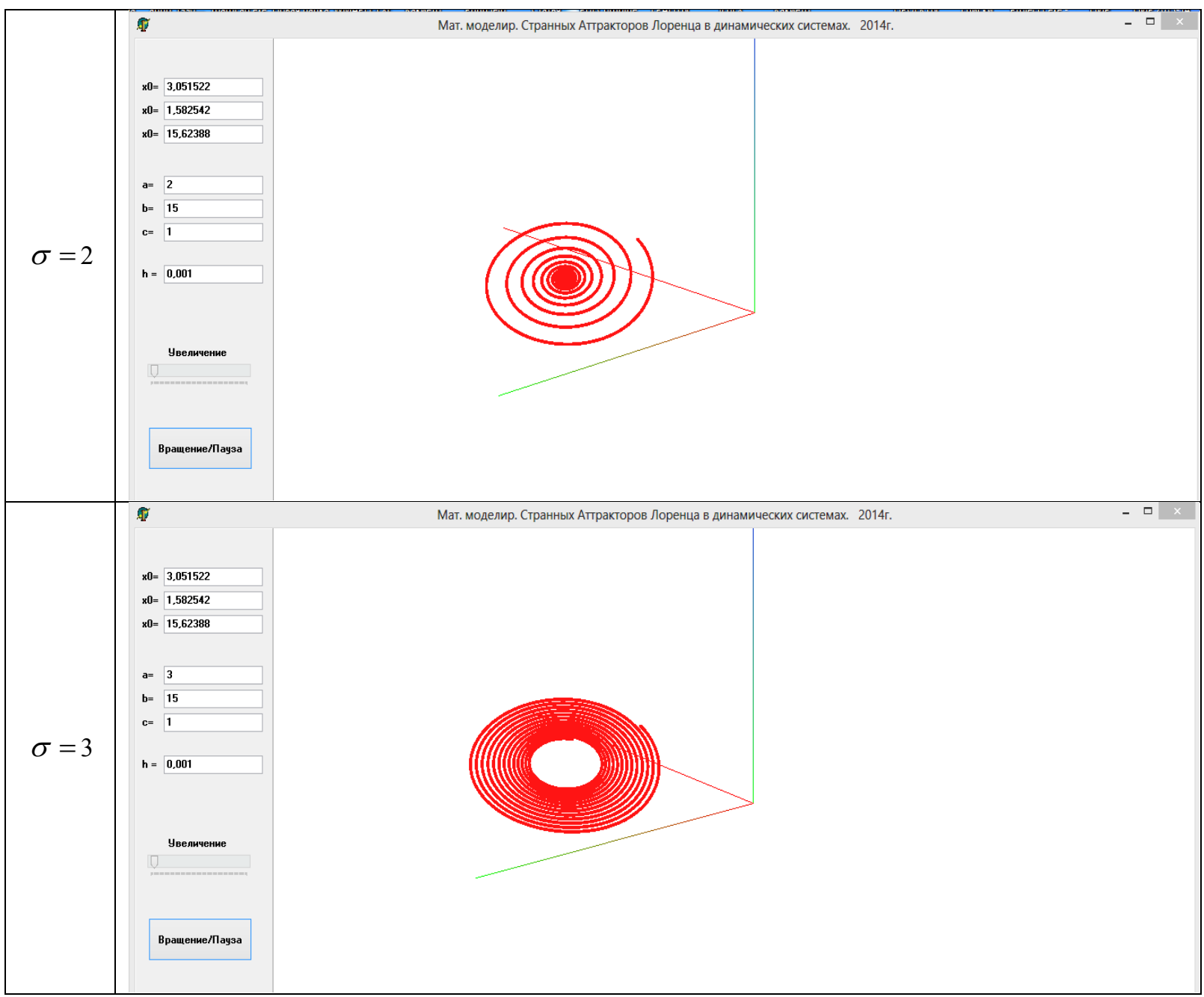


Impact Factor ISRA $($ India $)=\mathbf{1 . 3 4 4}$

Impact Factor ISI (Dubai, UAE) $=\mathbf{0 . 3 0 7}$

based on International Citation Report (ICR)
Impact Factor JIF $=\mathbf{1 . 5 0 0}$

Impact Factor GIF (Australia) $=\mathbf{0 . 3 5 6}$

Impact Factor SIS (USA) $=\mathbf{0 . 4 3 8}$

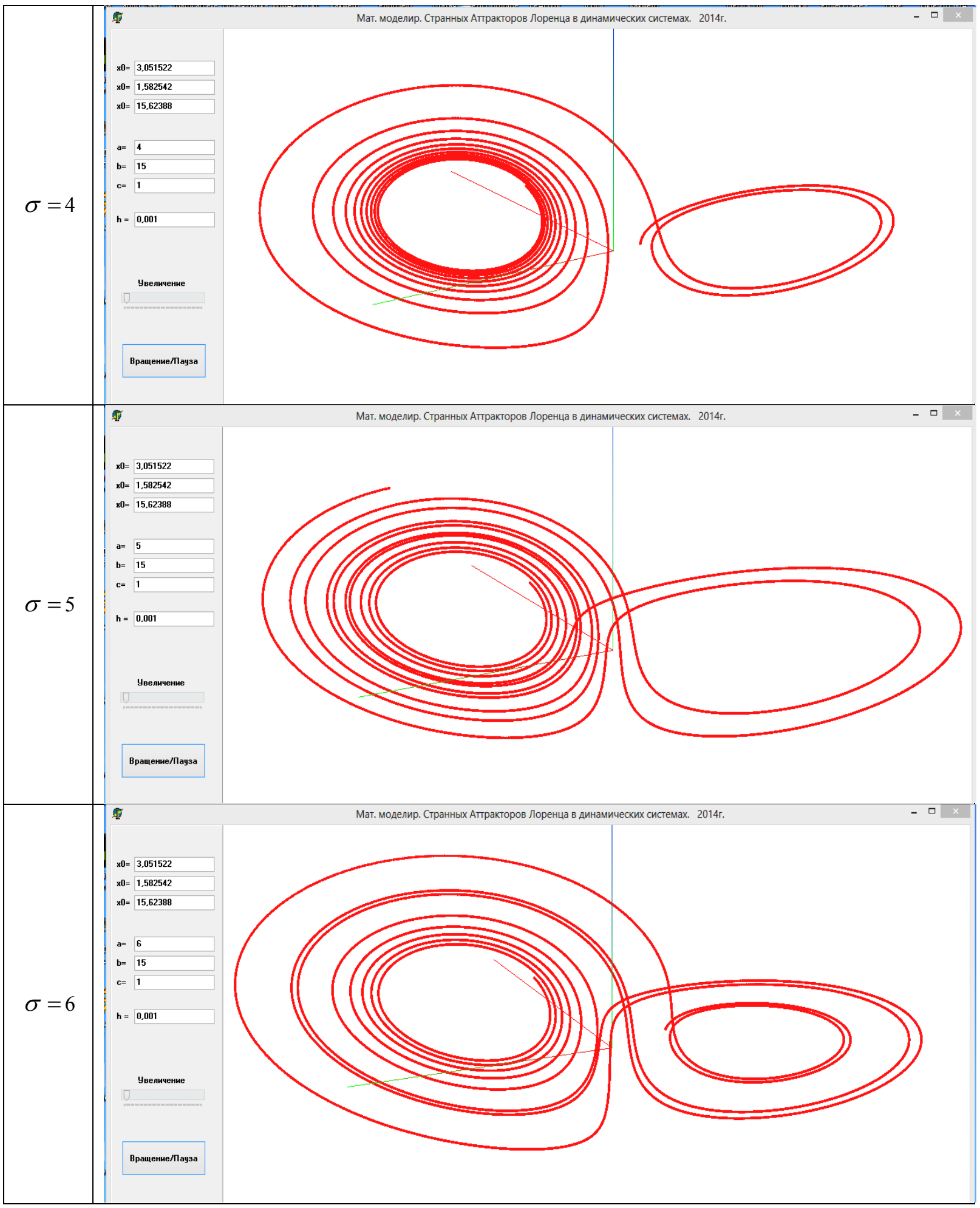


Impact Factor ISRA (India) $=\mathbf{1 . 3 4 4}$

Impact Factor ISI (Dubai, UAE) $=\mathbf{0 . 3 0 7}$

based on International Citation Report (ICR)
Impact Factor JIF $=\mathbf{1 . 5 0 0}$

Impact Factor GIF (Australia) $=\mathbf{0 . 3 5 6}$

Impact Factor SIS (USA) $=\mathbf{0 . 4 3 8}$

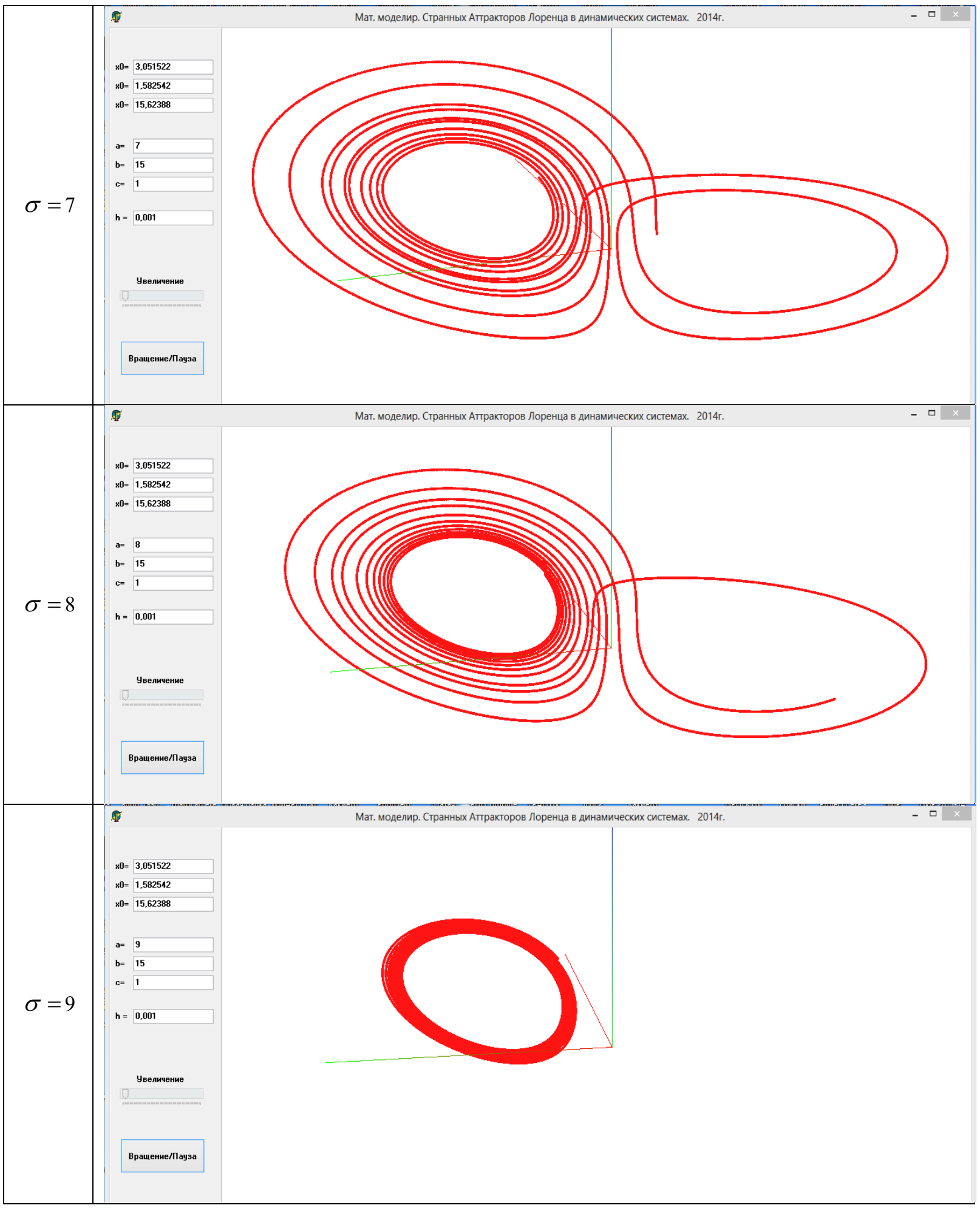


Impact Factor ISRA (India) $=\mathbf{1 . 3 4 4}$

Impact Factor ISI (Dubai, UAE) $=\mathbf{0 . 3 0 7}$

based on International Citation Report (ICR)
Impact Factor JIF $=\mathbf{1 . 5 0 0}$

Impact Factor GIF (Australia) $=\mathbf{0 . 3 5 6}$

Impact Factor SIS (USA) $=\mathbf{0 . 4 3 8}$

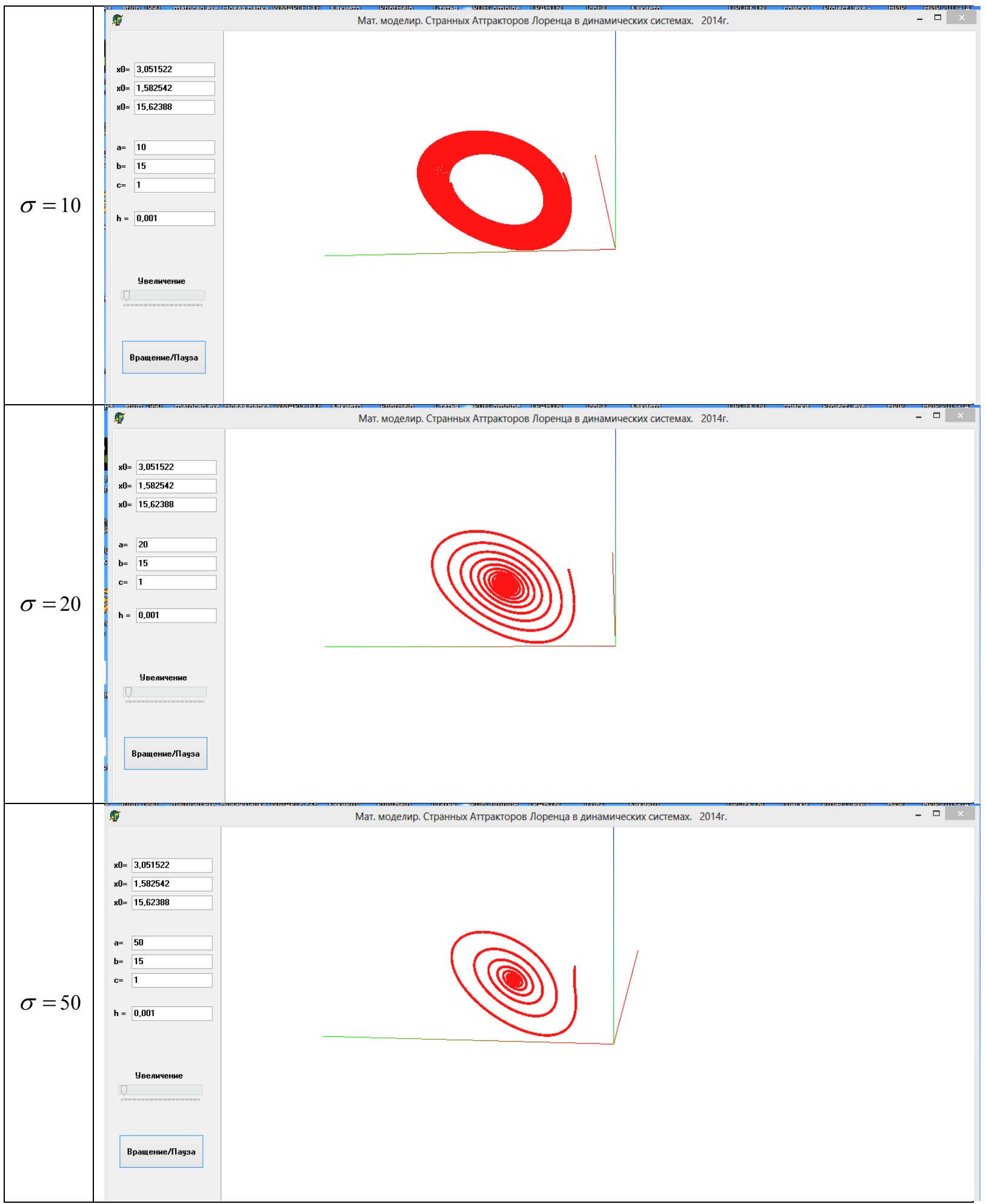



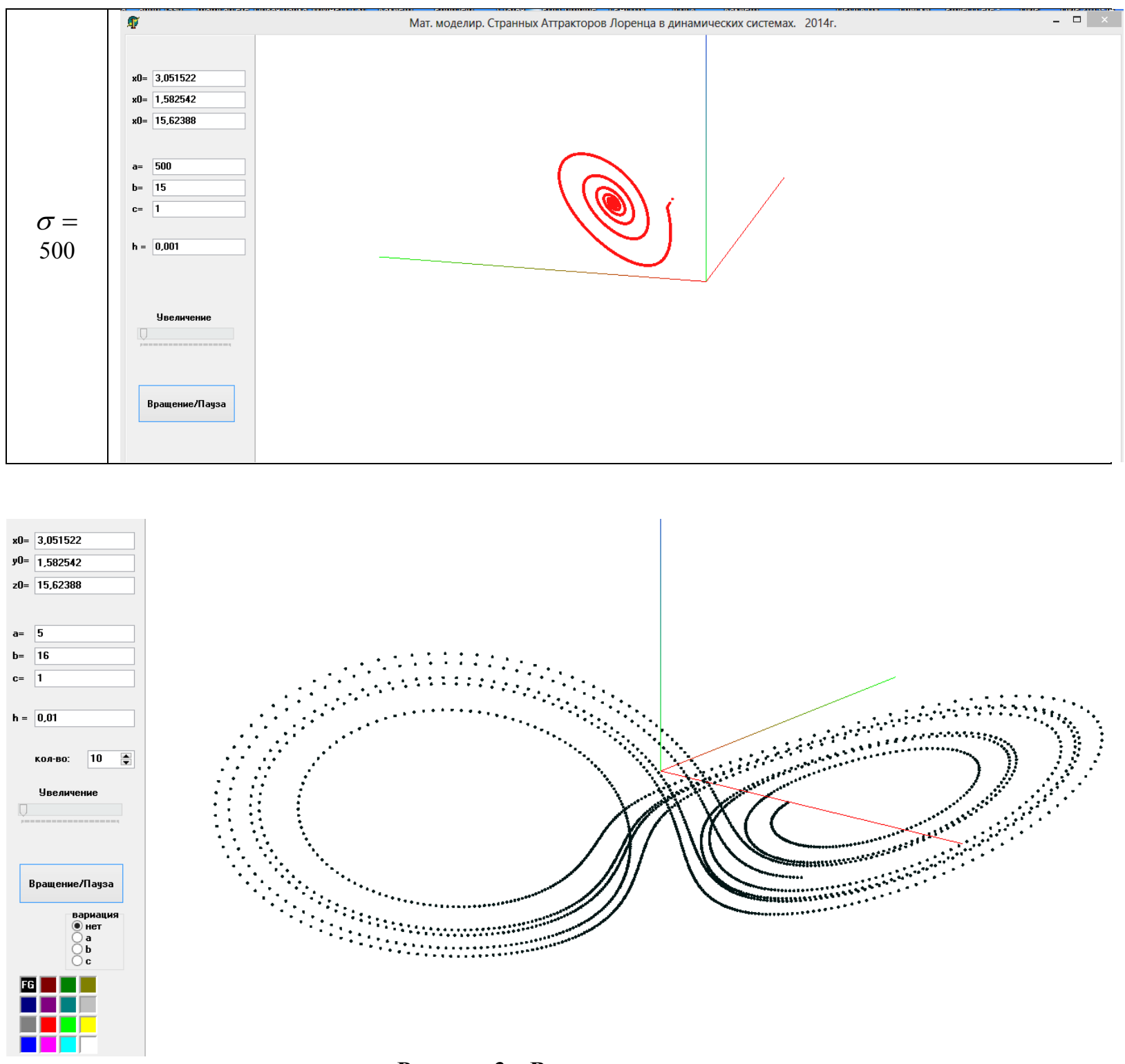

$\times 0=3.051522$

$y 0=1,582542$

$z 0=15,62388$

$a=5$

$b=16$

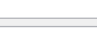

$h=0.01$

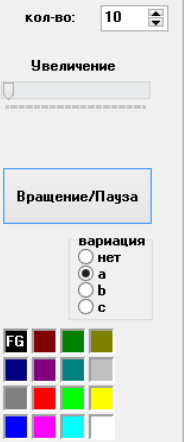

Рисунок 3 - Вариация отсутствует.

Рисунок 4 -Вариация по $\sigma$ 


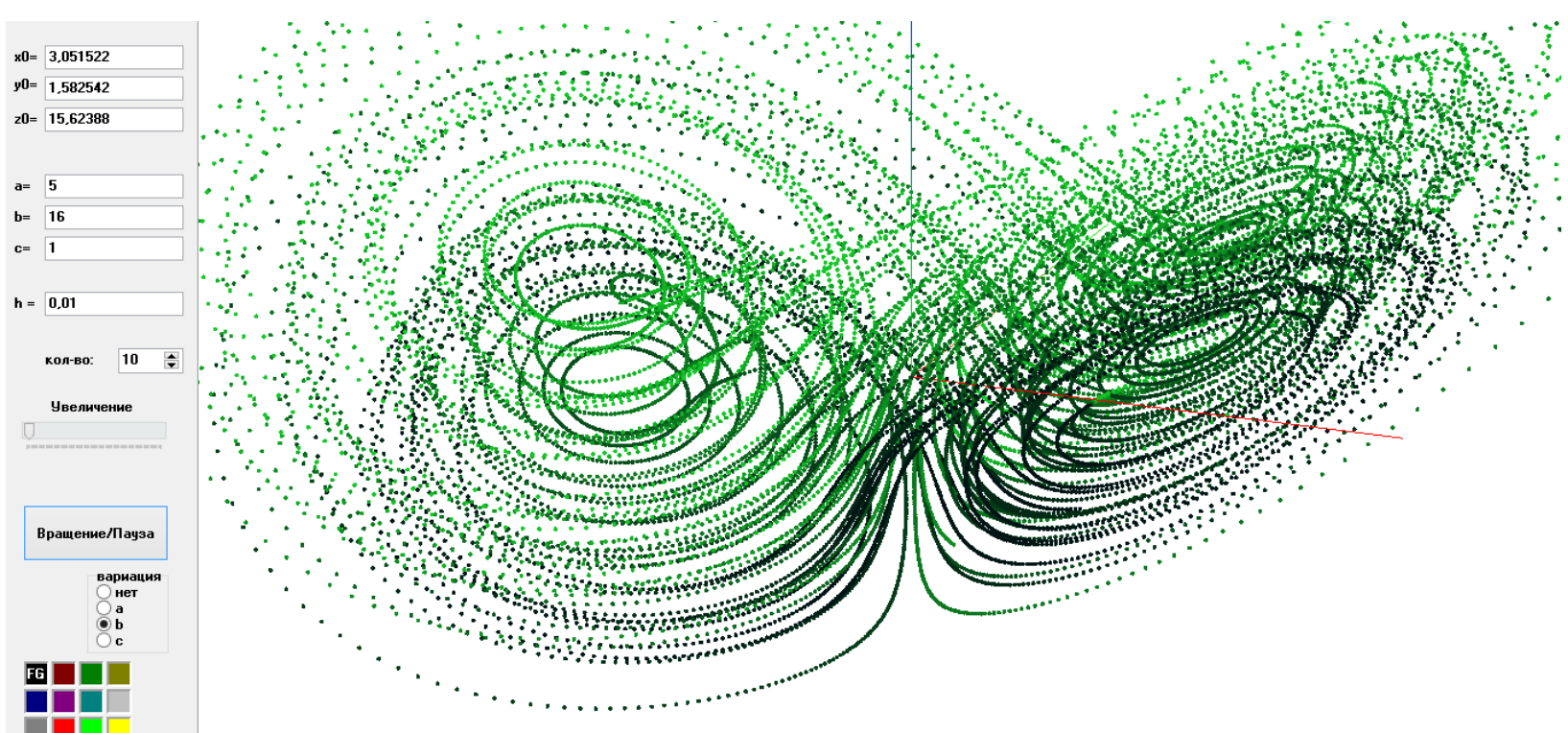

Рисунок 5 -Вариация по $r$.
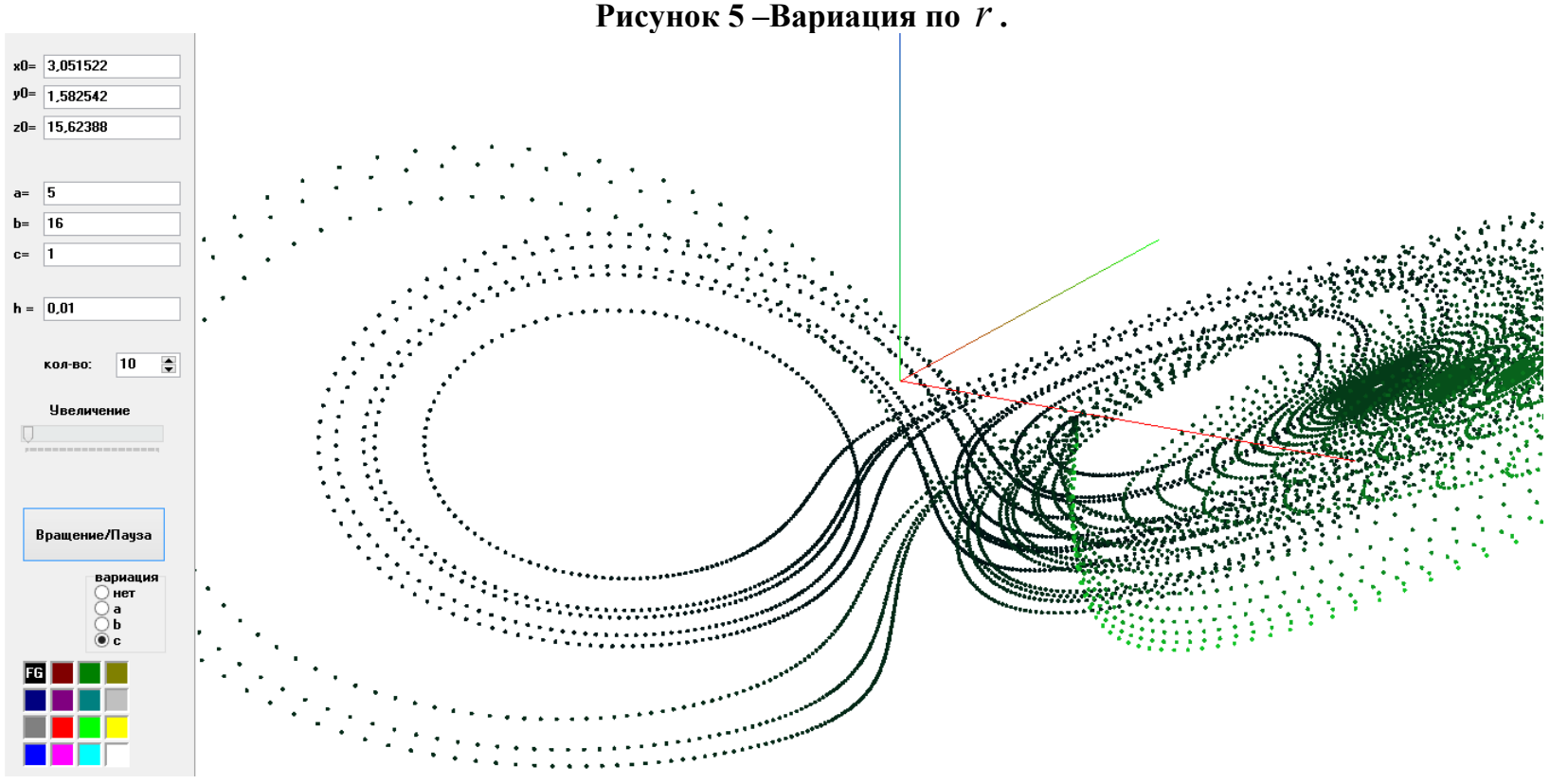

Рисунок 6 - Вариация по $b^{*}$.

Заключение.

Рассмотренная система дифференциальных уравнений приводит к решению в виде аттрактора Лоренца. Разработанная математическая модель и алгоритмы позволяют реализовать аттрактор Лоренца в 3D формате. Ниже приводится полный текст программы на Delphi, для реализации аттрактора.

Наиболее перспективным направлением дальнейшего исследования представляется:

- Исследование поведение аттрактора при различных изменении параметров для поиска точек бифуркации.
- Поиск решений системы в виде аттрактора, когда в левой части системы (1) стоят вторые производные функций.

- Поиск решений системы в виде аттрактора, когда в левой части системы (1) стоят $n$-е производные.

- Поиск таких решений системы (1) когда независимо от порядка производных функции правой части приводят к появлению решения в виде аттрактора. 


\section{Программа}

\section{Таблица 2}

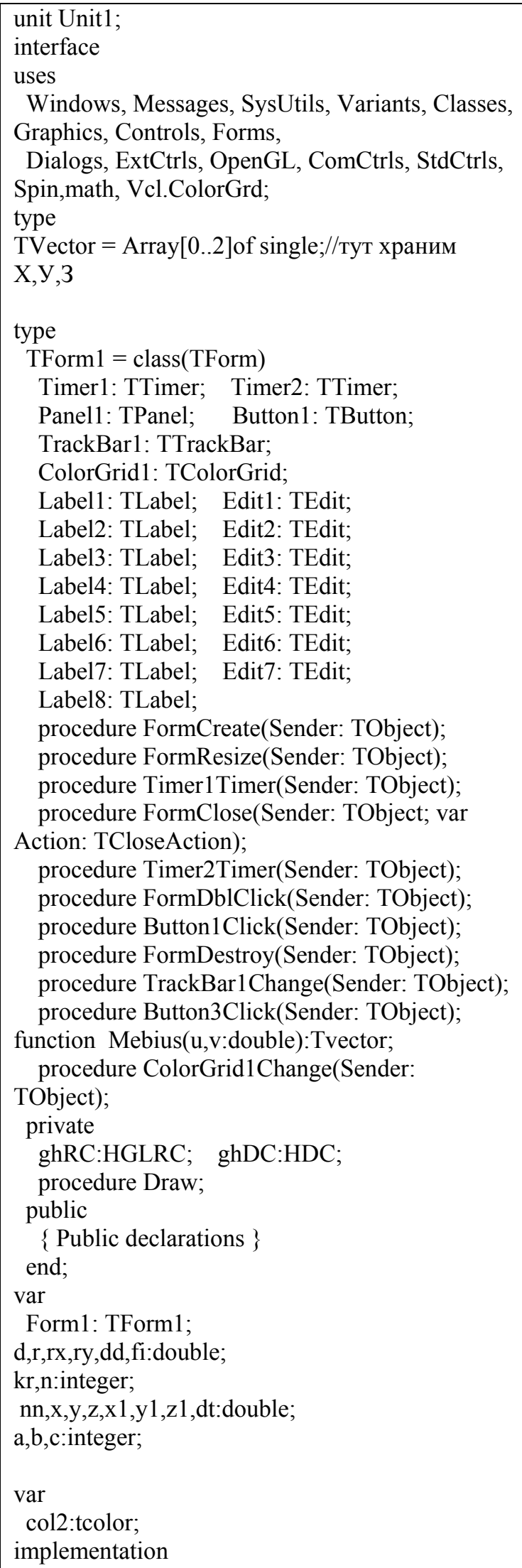

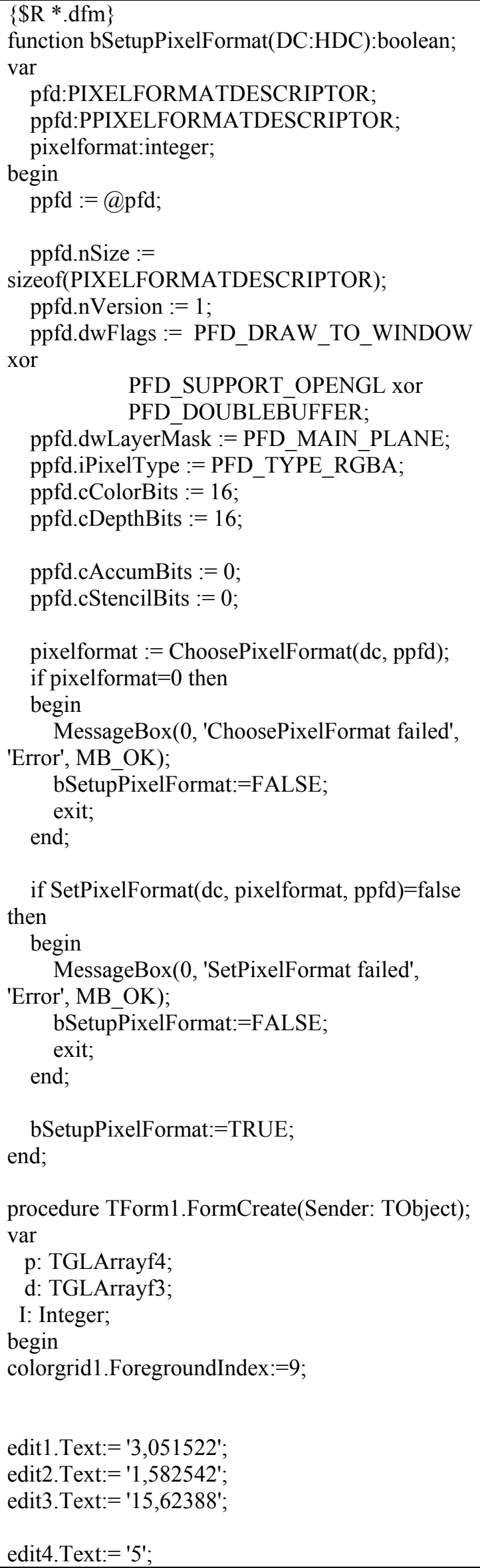




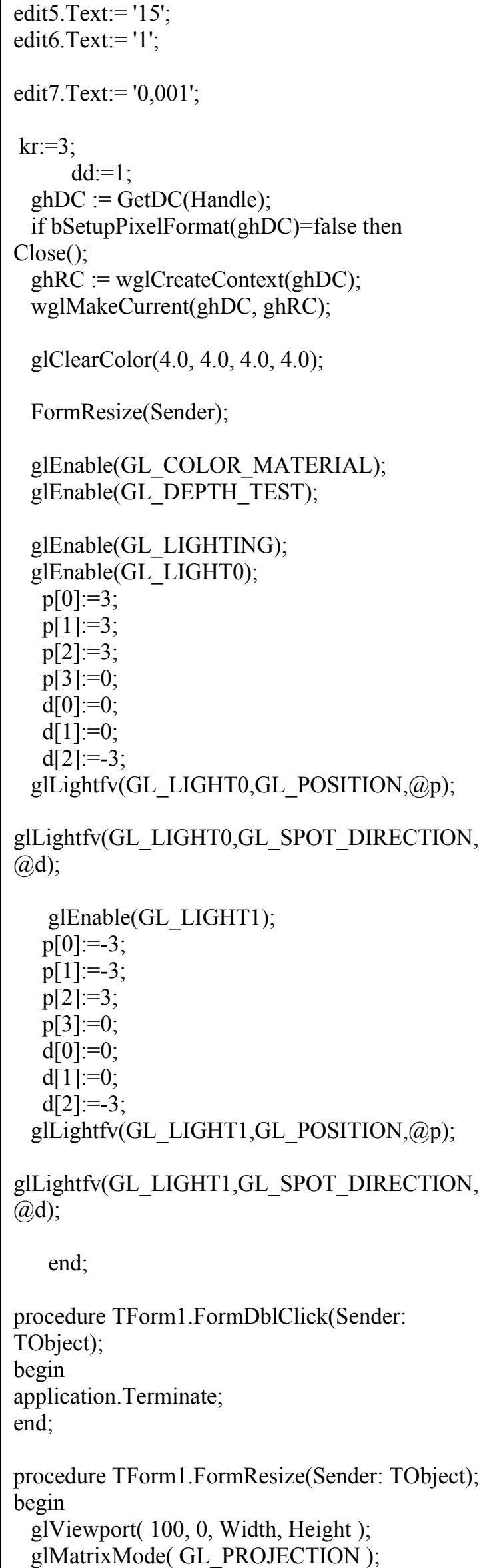

glLoadIdentity()

glOrtho(-kr/2, $\mathrm{kr} / 2, \quad-\mathrm{kr} / 2, \mathrm{kr} / 2, \quad 0,30)$;

gluLookAt( $2,2,2, \quad 0,0,0, \quad 0,0,10)$; glMatrixMode( GL_MODELVIEW ); end;

//• GL_POINTS каждая вершина задает координаты некоторой точки.

//• GL_LINES каждая отдельная пара вершин определяет отрезок; если задано //нечетное число вершин, то последняя вершина игнорируется.

procedure TForm1.Button1Click(Sender: TObject);

begin

Timer1.Enabled: $=$ not(Timer1.Enabled); if not(Timer1.Enabled) then exit;

Draw;

end;

procedure TForm1.ColorGrid1Change(Sender: TObject);

begin

col2:=ColorGrid1.ForegroundColor;

end;

procedure TForm1.Draw;

var i:integer;

h:double;

begin

glClear(GL_DEPTH_BUFFER_BIT xor

GL_COLOR_BUFFER_BIT);

glPolygonMode(GL FRONT AND BACK, $\{$ GL_FILL $\}$ GL_LINE);

if edit7.Text $<>$ " then $\mathrm{dt}:=$ strtofloat(edit7.Text); if edit1.Text $<>$ " then $\mathrm{x}:=$ strtofloat(edit1.Text); if edit2.Text $<>$ " then $\mathrm{y}:=$ strtofloat(edit2.Text); if edit3.Text $<>$ " then $z:=$ strtofloat(edit3.Text); if edit4.Text $<>$ " then $a:=$ strtoint(edit4.Text); if edit5.Text $<>"$ then $b:=$ strtoint(edit5.Text); if edit6.Text $<>$ " then $\mathrm{c}:=$ strtoint(edit6.Text);

glEnable(GL_POINT_SMOOTH); glPointSize(2); glBegin(GL_POINTS);

$\mathrm{nn}:=-10$;

while $n n<10$ do

begin

$\mathrm{nn}:=\mathrm{nn}+\mathrm{dt}$;

$$
\begin{aligned}
& \mathrm{x} 1:=\mathrm{x}+\mathrm{a}^{*}(-\mathrm{x}+\mathrm{y})^{*} \mathrm{dt} \\
& \mathrm{y} 1:=\mathrm{y}+\left(\mathrm{b}^{*} \mathrm{x}-\mathrm{y}-\mathrm{z}^{*} \mathrm{x}\right) * \mathrm{dt} \\
& \mathrm{z} 1:=\mathrm{z}+\left(-\mathrm{c}^{*} \mathrm{z}+\mathrm{x} * \mathrm{y}\right)^{*} \mathrm{dt} \\
& \mathrm{x}:=\mathrm{x} 1 \\
& \mathrm{y}:=\mathrm{y} 1
\end{aligned}
$$




z:= z1;
glcolor3f(Col2,Col2 shr 8+0.1,Col2 shr 16+0.1);
glVertex3d(x/10,y/10,z/10-1.2);
end;
glEnd();
glDisable(GL_POINT_SMOOTH);
glBegin(GL_LINE_STRIP);
glcolor3f(10,0,0);
glVertex3f(1,0,0);
glVertex3f(0,0,0);
glcolor3f( $(0,10,0) ;$
glVertex3f(0,1,0);
glVertex3f( $(0,0,0) ;$
glcolor3f( $(0,0,10) ;$
glVertex( $(0,0,2) ;$
glEnd;
glRotatef(-0.2, 0,0,1);
SwapBuffers(ghDC);
end;
procedure TForm1.Timer1Timer(Sender:
TObject);
begin
draw;

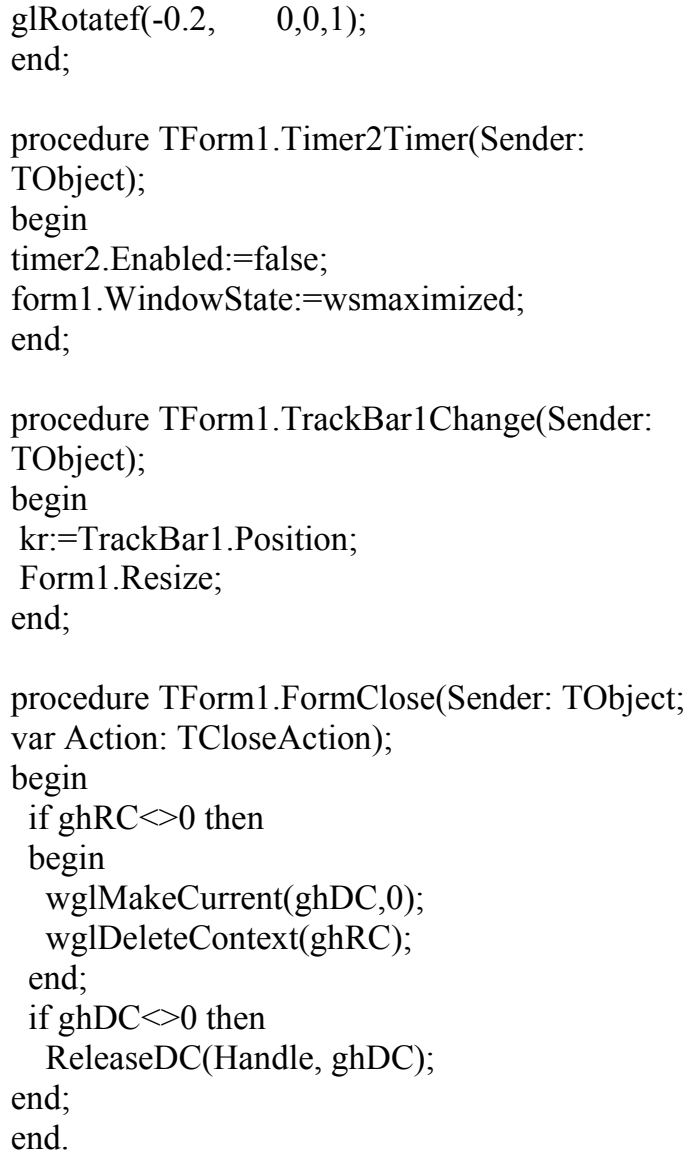

\section{References:}

1. (2014) Attraktor. Available: http://rfwiki.org/\%D0\%90\%D1\%82\%D1\%82\% D1\%80\%D0\%B0\%D0\%BA\%D1\%82\%D0\%B $\mathrm{E} \% \mathrm{D} 1 \% 80$

2. (2014) Atractor Poisson Saturne. Available: http://rfwiki.org/\%D0\%A4\%D0\%B0\%D0\%B9 \%D0\%BB:Atractor Poisson Saturne.jpg

3. Gorodetski A, Ilyashenko Y (1996) Minimal and strange attractors, International Journal of Bifurcation and Chaos, vol. 6, no. 6, pp. 11771183.

4. Gorodetskiy AS (2001) Minimal'nye attraktory i chastichno giperbolicheskie mnozhestva dinamicheskikh sistem. Diss. k. f.-m. n., MGU.

5. (2014) Stat'ya Dzh. Milnora «Attraktor», Scholarpedia.

6. (2013) Galereya samykh strannykh attraktorov. LENTA.RU. 28 mar 2013. Available: http://www.lenta.ru/photo/2013/03/28/visualizat ion/

7. (2013) Arkhivirovano iz pervoistochnika 4 apr 2013 http://www.webcitation.org/6FcS6HjdP
Available:
8. Nikul'chev EV (2007) Geometricheskiy metod rekonstruktsii sistem po eksperimental'nym dannym. Pis'ma v ZhTF. T. 33. Vyp. 6. S. 83-89.

9. Nikul'chev EV (2010) Identifikatsiya dinamicheskikh sistem na osnove simmetriy rekonstruirovannykh attraktorov $\mathrm{m}$. Available: http://nikulchev.ru/learnbook3.html

10. (2014) Attraktor Lorentsa. Available: http://rfwiki.org/\%D0\%90\%D1\%82\%D1\%82\% D1\%80\%D0\%B0\%D0\%BA\%D1\%82\%D0\%B E\%D1\%80 \%D0\%9B\%D0\%BE\%D1\%80\%D0 $\% \mathrm{~B} 5 \% \mathrm{D} 0 \% \mathrm{BD} \% \mathrm{D} 1 \% 86 \% \mathrm{D} 0 \% \mathrm{~B} 0$

11. Kuznetsov SP (2001) Lektsiya 3. Sistema Lorentsa; Lektsiya 4. Dinamika sistemy Lorentsa. Dinamicheskiy khaos (kurs lektsiy). Moscow: Fizmatlit.

12. Saltzman B (1962) Finite amplitude free convection as an initial value problem. Journal of the atmospheric science, No.7, pp. 329-341.

13. Lorents E (1981) Determinirovannoe neperiodicheskoe dvizhenie. Strannye attraktory. Moscow, pp. 88-116. 
14. (2014) Risuem prostye ob'ekty. Available: http://citforum.ru/programming/opengl/opengl $\underline{03 . s h t m l}$

15. Shevtsov AN, Beken BK, Talasbayev AA (2013) Study of parallel computations on delphi. ISPC Development of Applied Mathematics, 30.05.2013, Taraz, Kazakhstan. ISJ Theoretical \& Applied Science 5(1): 28-36. doi: http://dx.doi.org/10.15863/TAS.2013.05.1.6

16. Shevtsov AN, Asanbayeva MM (2013) About one algorithm of recognition sharply distinguished objects. ISPC Development of Applied Mathematics, 30.05.2013, Taraz, Kazakhstan. ISJ Theoretical \& Applied Science 5(1): 41-47. doi: http://dx.doi.org/10.15863/TAS.2013.05.1.8

17. Shevtsov AN, Asanbayeva MM (2013) ALGORITHM OF ALLOCATION OF BORDERS OF OBJECT. ISPC Development of Applied Mathematics, 30.05.2013, Taraz, Kazakhstan. ISJ Theoretical \& Applied Science 5(1): 52-58. doi: http://dx.doi.org/10.15863/TAS.2013.05.1.10

18. Kestelman VN, Shevtsov AN, Nadirbekova AS (2013) ON SOME SOLUTIONS OF FREDHOLM EQUATIONS 2 KIND SQUARE METHOD.. «World of Science», ISPC, 30.06.2013, Hamburg, Germany. ISJ Theoretical \& Applied Science 6(2), pp.1-15. doi: http://dx.doi.org/10.15863/TAS.2013.06.2.1

19. Zhunisbekov S, Jönsson A, Shevtsov AN ABOUT SOME CLOUD CHI-SQUARE CRITERION PEARSON. ISPC «Theory and Practice», 30.08.2013, Munich, Germany. ISJ Theoretical \& Applied Science 8(4), pp.1-23. doi: http://dx.doi.org/10.15863/TAS.2013.08.4.1

20. Zhunisbekov S, Shevtsov AN ALGORITHMS FOR CALCULATING THE PROPERTIES AND BEHAVIOR ANALYSIS OF FRACTIONAL-LINEAR MAPPINGS IN DELPHI. ISPC «Results \& Perspectives», 30.09.2013, Florence, Italy. ISJ Theoretical \& Applied Science 9(5), pp.1-13. doi: http://dx.doi.org/10.15863/TAS.2013.09.5.1

21. Shevtsov AN, Nietbaev AA, Perneshova BK ALGORITHMS OF ANALYSIS OF THE SPECTRAL DISTRIBUTION. ISPC «Results \& Perspectives», 30.09.2013, Florence, Italy. ISJ Theoretical \& Applied Science 9(5), pp.18-50. doi: http://dx.doi.org/10.15863/TAS.2013.09.5.3

22. Shevtsov AN, Keulimzhayeva ZA, Alpysbayev EA (2013) ABOUT SOME MODELS OF
POLYMER MACROMOLECULE. Materials of the ISPC «Applied scientific research», 30.11.2013, Belgrade, Serbia. ISJ Theoretical \& Applied Science 11(7), pp.26-40. doi: http://dx.doi.org/10.15863/TAS.2013.11.7.4

23. Zhunisbekov S, Shevtsov AN (2013) ABOUT ONE MODEL OF THE PROCESS OF CRYSTALLIZATION. ISPC «The results of scientific research in 2013», 30.12.2013, Neoplanta, Serbia. ISJ Theoretical \& Applied Science 12(8), pp.1-4. doi: http://dx.doi.org/10.15863/TAS.2013.12.8.1

24. Shevtsov AN (2013) ABOUT SOME ALGORITHMS FOR CONSTRUCTING THREEDIMENSIONAL DYNAMICAL MODELS. ISPC «The results of scientific research in 2013», 30.12.2013, Neoplanta, Serbia. ISJ Theoretical \& Applied Science 12(8), pp.9-16. doi: http://dx.doi.org/10.15863/TAS.2013.12.8.3

25. Shevtsov AN (2014) SOME QUESTIONS SIMULATION OF INTERACTIVE DYNAMIC SYSTEMS. ISPC «Economy, technology, education and prospects for 2014», 30.01.2014, Malmö, Sweden. ISJ Theoretical \& Applied Science 1(9), pp.5-22. doi: http://dx.doi.org/10.15863/TAS.2014.01.9.2

26. Shevtsov AN (2014) SOME ALGORITHMS OF FRACTAL COMPRESSION. ISPC «Scientific technologies of the future», 28.02.2014, Linköping, Sweden. ISJ Theoretical \& Applied Science 2(10), pp.12-28. doi: http://dx.doi.org/10.15863/TAS.2014.02.10.3

27. Zhunisbekov S, Shevtsov AN, Keulimzhayeva ZA (2014) ON THE PROBLEM OF MODELING OF MACROMOLECULES OF POLYMERS. ISPC Integration of Science \& Education, 30.03.2014, Gothenburg, Sweden. ISJ Theoretical \& Applied Science 3(11), pp.3562. doi: http://dx.doi.org/10.15863/TAS.2014.03.11.7

28. Kestelman VN, Shevtsov AN, Akhmetkaliyeva AK (2014) THREE-DIMENSIONAL TOPOLOGICAL CODING WITH A PRIVATE KEY ON THE MOBIUS STRIP. ISPC Computer technologies in science, 30.04.2014, Valencia, Venezuela. ISJ Theoretical \& Applied Science 4 (12), pp.49-72. doi: http://dx.doi.org/10.15863/TAS.2014.04.12.9 